\title{
A GENERALIZATION OF A THEOREM ON NATURALLY REDUCTIVE HOMOGENEOUS SPACES
}

\author{
OLDR̆ICH KOWALSKI AND LIEVEN VANHECKE
}

\begin{abstract}
We prove that a homogeneous Riemannian manifold all of whose geodesics are orbits of one-parameter subgroups of isometries has volumepreserving local geodesic symmetries.
\end{abstract}

Let $(M, g)$ be a Riemannian manifold. $(M, g)$ is said to be naturally reductive $[\mathbf{6}]$ if there is a connected Lie group $G$ of isometries acting transitively and effectively on $M$ such that $M=G / H$ ( $H$ being the isotropy subgroup of $G$ at some point of $M)$ is a reductive homogeneous space with respect to the decomposition $\mathfrak{g}=\mathfrak{m}+\mathfrak{h}$ of the Lie algebra $\mathfrak{g}$ of $G$ and moreover

$$
\left\langle[X, Y]_{\mathfrak{m}}, Z\right\rangle+\left\langle[X, Z]_{\mathfrak{m}}, Y\right\rangle=0 \quad \text { for } X, Y, Z \in \mathfrak{m} .
$$

Here $\langle$,$\rangle denotes the inner product on \mathfrak{m}$ induced by the metric $g$. D'Atri and Nickerson $[\mathbf{1}, \mathbf{2}]$ have proved

THEOREM A. Let $(M, g)$ be a naturally reductive homogeneous space. Then

(a) all the local geodesic symmetries are volume-preserving;

(b) the characteristic polynomial $P(\lambda)=\operatorname{det}\left(g_{i j}-\lambda \delta_{i j}\right)$ of the metric tensor $g$ with respect to any system of normal coordinates centered at a point of the manifold is preserved under the local geodesic symmetry centered at the same point.

In $[\mathbf{4}, \mathbf{5}]$ A. Kaplan introduced the notion of a generalized Heisenberg group and proved that these spaces are naturally reductive if and only if the center of the corresponding algebra has dimension 1 or 3 . Moreover the following is true.

Proposition B. Let $M$ be a generalized Heisenberg group. Then

(a) all the local geodesic symmetries are volume-preserving;

(b) if $\operatorname{dim} M=6$ (two-dimensional center), then $P(\lambda)$ is preserved under the corresponding local geodesic symmetry at each point.

(a) is proved in [5] and (b) in [9].

Next, let $(M, g)=G / H$ where $G$ is the connected component $I_{0}(M)$ of the full isometry group of $M$. Then, $(M, g)$ is said to be a commutative space if the algebra $D(G / H)$ of invariant differential operators on $G / H$ is commutative. The 6-dimensional Heisenberg group provides an example and hence, (b) of Proposition $\mathrm{B}$ is a corollary of the following theorem.

Received by the editors July 27,1983 .

1980 Mathematics Subject Classification. Primary 53C30, 53C20.

Key words and phrases. Naturally reductive homogeneous spaces, commutative spaces, generalized Heisenberg groups, volume-preserving geodesic symmetries, orbits of one-parameter subgroups. 
ThEOREM C [7]. Let $(M, g)$ be a commutative space. Then, the volume and also the characteristic polynomials $P(\lambda)$ are preserved under local geodesic symmetries.

The main purpose of this paper is to prove two theorems which provide new proofs of Theorem A and (b) of Proposition B, and which at the same time generalize both.

THEOREM 1. Let $(M, g)=G / H$ be a homogeneous Riemannian manifold such that all its geodesics are orbits of one-parameter subgroups of $G$. Then $(M, g)$ is a space with volume-preserving geodesic symmetries.

Proof. For any fixed $m \in M$ and a normal neighborhood $U_{m}$, define the normal volume function $\theta_{m}: U_{m} \rightarrow \mathbf{R}$ by

$$
\theta_{m}(p)=\left.\omega\left(\frac{\partial}{\partial x^{1}}, \ldots, \frac{\partial}{\partial x^{n}}\right)\right|_{p}, \quad p \in U_{m}
$$

where $\omega$ is a (local) Riemannian volume form defined near $m$ and $\left(x^{1}, \ldots, x^{n}\right)$ is a positively oriented system of normal coordinates centered at $m$. It is easy to see that $\theta_{m}(p)$ does not depend on the choice of $\omega$ and the choice of the normal coordinates.

Further we have, for each isometry $\varphi \in I(M)$,

$$
\theta_{\varphi(m)}(\varphi(p))=\theta_{m}(p)
$$

and it is proved in $[3$, pp. 156-157] that

$$
\theta_{m}(p)=\theta_{p}(m)
$$

for any $p$ sufficiently close to $m$.

Finally, let $-p$ denote the antipodal point of $p$ with respect to the center $m$. Then, $M$ is a space with volume-preserving geodesic symmetries if and only if for all $m$ and all $p$ near $m$ we have

$$
\theta_{m}(-p)=\theta_{m}(p)
$$

To prove the required result, consider the geodesic $\gamma(t)$ such that $\gamma(0)=m$, $\gamma(\tau)=p, \gamma(-\tau)=-p$ for some $\tau>0$. According to our assumption, there is an $A \in \mathfrak{g}$ such that $\gamma(t)=(\exp t A)(m)$ for all $t \in \mathbf{R}$. Put $\varphi=\exp (-\tau A)$. Then we have $\varphi(m)=-p, \varphi(p)=m$. Hence (2) and (3) imply (4).

THEOREM 2. Under the hypothesis of Theorem 1, the characteristic polynomial $P(\lambda)$ of the metric tensor with respect to any normal coordinate system is preserved under the corresponding local geodesic symmetry.

PROOF. We use the same argument as before; instead of (2) we have the invariance of $P(\lambda)$ with respect to all isometries and instead of $(3)$ we use the formulas

$$
\mu_{k m}(p)=\mu_{k p}(m), \quad k=1, \ldots, n,
$$

from [10], where the $\mu_{k m}$ denote the coefficients of $P(\lambda)$ with reference point $m$.

Theorem $\mathrm{A}$ follows now at once since all geodesics of a naturally reductive homogeneous space are orbits of one-parameter subgroups. The same property holds for the six-dimensional generalized Heisenberg group (see $[\mathbf{5}, \mathbf{9}]$ ) and hence (b) of 
Proposition B also follows. In fact, Theorem 2 applies to the broader subclass of generalized Heisenberg groups determined explicitly by C. Riehm (see [5, p. 42]).

REMARK. It can be seen from $[\mathbf{5}, \mathbf{8}]$ that there are generalized Heisenberg groups (with four-dimensional center) whose geodesics are not all orbits of oneparameter subgroups but which are still commutative spaces. On the other hand, there are also generalized Heisenberg groups (for example with five-dimensional center) whose geodesics are all orbits of one-parameter subgroups but it is not known to us whether these spaces are commutative or not. Yet, we believe that the property "geodesics are orbits of one-parameter subgroups" might well imply the commutativity for the class of all Riemannian homogeneous manifolds.

\section{REFERENCES}

1. J. E. D'Atri and H. K. Nickerson, Geodesic symmetries in spaces with special curvature tensor, J. Differential Geom. 9 (1974), 251-262.

2. J. E. D'Atri, Geodesic spheres and symmetries in naturally reductive spaces, Michigan Math. J. 22 (1975), 71-76.

3. A. L. Besse, Manifolds all of whose geodesics are closed, Ergebnisse der Mathematik, Vol. 93, Springer-Verlag, Berlin and New York, 1978.

4. A. Kaplan, Riemannian nilmanifolds attached to Clifford modules, Geom. Dedicata 11 (1981), 127-136.

5. __ On the geometry of groups of Heisenberg type, Bull. London Math. Soc. 15 (1983), 35-42.

6. S. Kobayashi and K. Nomizu, Foundations of differential geometry. II, Interscience, New York, 1969.

7. O. Kowalski and L. Vanhecke, Opérateurs différentiels invariants et symétries géodésiques préservant le volume, C. R. Acad. Sci. Paris Sér. I Math. 296 (1983), 1001-1003.

8. C. Riehm, The automorphism group of a composition of quadratic forms, Trans. Amer. Math. Soc. 269 (1982), 403-414.

9. F. Tricerri and L. Vanhecke, Homogeneous structures on Riemannian manifolds, London Math. Soc. Lecture Note Series 83, Cambridge Univ. Press, London, 1983.

10. L. Vanhecke, The canonical geodesic involution and harmonic spaces, Ann. Global Analysis and Geometry 1 (1983), 131-136.

Faculty of Mathematics and Physics, Charles University, Sokolovská 83, 18600 Praha, CZechoslovakia

Department of Mathematics, Katholieke Universiteit Leuven, CelestiJNENLAAN 200B, B-3030 LEUVEN, BELGIUM 